\title{
PARTICIPACIÓN COMUNITARIA EN SALUD: LA EXPERIENCIA DE LOS GRUPOS DE SALUD DE LA ZONA OESTE
}

\author{
M. Cristina Di Silvestre Paradizo
}

\section{INTRODUCCIÓN}

En la actualidad la participación social entendida como proceso en el cual los actores sociales toman parte en la identificación y definición de sus problemas y en la gestión de las soluciones, constituye un tema de interés nacional que va de la mano del proceso de democratización de nuestro país. No obstante lo cual esto no significa que en el pasado no se manifestó de distintas formas y en diferentes ámbitos. Entre los años 1920 y 1950 se produce en Chile una creciente concentración de población en la capital debido a la crisis petrolera. Esta población que demanda trabajo, vivienda, educación, comienza a expandirse hacia la periferia, constituyendo anillos alrededor de Santiago. Se desarrollan acciones de los pobladores, especialmente por demandas de viviendas, la "operación sitio" fue establecida bajo los principios de autoconstrucción.

En la década del 50, en América Latina y el Caribe, se promovieron muchas experiencias con el objeto de provocar un cambio cultural planificado. En esa época ya se hablaba de "desarrollo de la comunidad". A la luz del análisis de la situación de los países en desarrollo, éstos fueron considerados como formando parte de un mundo tradicional, con características y estilos de vida que limitaban el progreso y desarrollo. Se les supuso carentes de capacidades y recursos a fin de modernizar sus sociedades. Se conforma el paradigma de la modernización, pero desde la perspectiva de los países desarrollados y modernos. Se postula que mediante la implementación de ciertos programas, como el Plan Marshall y la Alianza para el Progreso y un conjunto de planes financiados por organismos internacionales (FAO, UNICEF, AID, IDCR, VHO/PAHO, MILBANK FOUNDATION, etc.) se podría sacar del subdesarrollo a estos países conjuntamente con una participación de la comunidad en estos planes. Se decía que cada grupo debía intervenir y sumar sus recursos para brindar su aporte en un esfuerzo de desarrollo nacional.

Específicamente en Chile, en el área de la Salud, La creación del Servicio Nacional de Salud en 1952, dio lugar a la formulación de Programas de Desarrollo y Organización de las Comunidades que habían sido propuestos por la Organización Mundial de la Salud. Se definía en aquel entonces la participación como "un proceso por el cual el propio pueblo participa en la planificación y en la realización de programas que se destinan a elevar su nivel de vida" (Citado por Hevia, 1991, pág. 4).

La participación comunitaria es visualizada como un imperativo toda vez que ningún país de América Latina y el Caribe contaba con recursos suficientes para implementar el tipo de reformas propuestas por los organismos internacionales (Ugalde: 1985). En Chile, durante el gobierno del presidente Frei se crearon los Comités o Consejos de Salud (Decreto № 250, 1967). Estos consejos constituyeron un esfuerzo por promover la participación de la comunidad en la búsqueda de soluciones a sus problemas de salud. Complementando esta iniciativa en el año 1969, se propuso la política de acción comunitaria. Así el Servicio Nacional de Salud contribuyó a iniciar la articulación entre equipos de Salud y comunidad mediante trabajos colaborativos. Una impor-

*Académica, Departamento de Sociología, Universidad de Chile-Universidad de la República. 
tante limitante de los esfuerzos de esa época reside en que las propuestas y programas implementados tenían su origen no en la comunidad beneficiada, sino en un grupo externo a ella. Al respecto, Ugalde (1985) formula una crítica interesante cuya exposición es pertinente en este contex to. Estc autor postula que los Programas de Participación Comunitaria han estado basados en dos supuestos errados:

1. La creencia de que los valores tradicionales de los pobres constituyen el principal obstáculo para el desarrollo.

2. "La idea de que el pobre es incapaz de organizarse ellos mismos" (Ugalde, p.7).

Más aún este autor agrega que el sistema de valores de campesinos y pobres no ha sido comprendido por los expertos de las Agencias Internacionales, a tal punto que se ha desconocido muchas de las características de "modernidad" de estos sectores sociales tales como: la ayuda mutua, la propiedad comunal de la tierra, la cooperación, el igualitarismo, elementos todos que favorecen una acción colectiva efectiva.

A pesar de la crítica anterior y de las de otros autores y de los mismos actores sociales, ya en la década del 70 la concepción de la participación de la comunidad comenzó a visualizarse bajo otra óptica. Es así como en la Primera Conferencia Internacional sobre Desarrollo de la Comunidad realizada en Santiago de Chile en julio de 1970, se postula como eje fundamental de desarrollo de la Comunidad, la incorporación a éste de los sectores populares, mediante su participación organizada en las fases de definición y acción en torno a los problemas que la aquejan. Bajo estos postulados es que en 1971, durante el Gobierno del Presidente Allcnde se elaboró un marco legal a la Constitución de los Consejos Locales y Consejos Paritarios de Salud a nivel de Establecimientos (consultorios, hospitales) y Área de Salud en todo el Servicio Nacional de Salud a través del país (Hevia, 1991).

Según estadísticas de esa época, en 1970 había 80 Consejos Comunitarios que aumentaron a 269 Consejos Locales y 207 Consejos Paritarios a fines de 1972.

Los Consejos Locales se caracterizaron como entidades democráticas en que concurrían representantes de los trabajadores de la Salud y de todas las Organizaciones Comunitarias del área de acción del respectivo consejo. Estos representantes eran convocados por la autoridad de salud local.

Los trabajos llevados a cabo tenían un carácter autónomo, en el sentido que eran definidos a nivel local (Hevia, 1991).

Distintos fueron los esfuerzos llevados a cabo en América Latina con el objeto de lograr una Participación Comunitaria en Salud. Las distintas experiencias y modalidades de expresión de esta participación recibieron diferentes rótulos: Voluntariado Comunitario, Comités locales de Salud, Consejos Locales de Desarrollo Comunal y Brigadas de Salud. Tal fue la importancia que logró la participación comunitaria en salud que en 1977, en la Cuarta reunión de los ministros de salud (Washington EE.UU.) la participación comunitaria pasó a constituirse en una estrategia básica para lograr ampliar la cobertura de los servicios de salud. Se plantea la necesidad de colaboración entre instituciones gubernamentales y comunidad.

A pesar que fueron muchos los intentos de establecer un trabajo conjunto, la forma en que éste tomó curso fue muy resistida y criticada toda vez que se caracterizó por ser autoritaria, verticalista y manipulativa (Corsaps, 1991). En consecuencia, la participación comunitaria continuó siendo más bien un propósito que un hecho concreto.

Como desafiando los fracasos y resistencias, la idea de participación comunitaria continuó estando vigente y siendo un tema recurrente en los Organismos Internacionales; La Organización Mundial de la Salud en su declaración de Alma Ata (Rusia, 1978) postuló como objetivo "salud para todos en el año 2000”. En la consecución de este objetivo la participación comunitaria asume 
un lugar preponderante. Se plantea que sólo mediante la participación de la comunidad es posible alcanzar una vida saludable para los sectores pobres y marginados del acceso a la salud. En esta declaración como en los lineamientos planteados por documentos de la Organización Mundial de la Salud publicados posteriormente se formuló la necesidad de la participación comunitaria no sólo en la identificación y definición de sus problemas de salud, sino en la planificación y gestión de las actividades para lograr solución a los mismos (Documento CD 33/14, 1988, "Strategies for Health for all by the year 2000", Génova, 1979).

A pesar de que ha transcurrido más de una década desde la declaración de Alma Ata, la participación comunitaria en salud no ha logrado aún establecerse. Este hecho está estrechamente ligado al contexto histórico, político y socioeconómico de los países de la América Latina y el Caribe. En general se ha producido una falta de respuesta por parte de las autoridades a las demandas de la comunidad, problemas en la implementación de la organización y coordinación entre los equipos de salud y la comunidad, persevera la forma paternalista y asistencial en la resolución de los problemas de salud, temor en las instituciones gubernamentales a abrir paso a la participación de la gente porque se confunde con bandera política toda vez que la salud es una de las dimensiones de la calidad de vida de las personas. Por ende se observa una falta de voluntad política para dar curso a la democratización de las instancias que permitan la participación comunitaria.

\section{ANTECEDENTES DE LA FORMACIÓN DE LOS GRUPOS DE SALUD DE LA ZONA OESTE: UNA EXPERIENCIA DE PARTICIPACIÓN COMUNITARIA EN SALUD}

En el año 1975, Ia Iglesia Católica organiza una entidad ecuménica que recibe el nombre de "Comité por la Paz en Chile", Cuyo objetivo fue prestar apoyo jurídico y de salud a las víctimas de violaciones a los derechos humanos.

Los grupos de salud surgen en el marco de la Iglesia Católica y el Comité por la Paz en Chile. Se constituye un equipo de personas profesionales de la salud que provenían del área estatal y que estaban por salir del país por problemas políticos. Este equipo se dedicó a elaborar un Programa de Salud orientado a dos áreas de trabajo:

1) Asistencia: atención médica y dental a víctimas de la represión, atención maternal, control de salud de niños en comedores infantiles, atención de salud familiar.

2) Capacitación: a cesantes, comunidades cristianas y madres asistentes a comedores infantiles acerca de higiene personal y ambiental y sobre pediculosis y sarna. La labor del equipo de salud se llevaba a cabo con la colaboración de médicos y convenios con laboratorios clínicos. A fin de detectar personas potencialmente necesitadas de servicios de salud, el programa formó una "red de responsables en salud". El equipo visualizó la posibilidad de formar madres a fin de que ellas mismas fueran las portadoras de la información dirigida a niños y padres asistentes a los Comedores Infantiles. Surgen así los llamados Grupos de Salud Poblacional.

Estas madres participantes tenían un efecto multiplicador en el sentido de que también les correspondía capacitar a los pobladores en problemas de salud.

El equipo técnico de salud también realizó una labor de eđucación para la salud. Con este propósito se elaboraron programas con contenidos de fácil comprensión y en lenguaje familiar para la población objetivo. Los grupos se fueron consolidando, la participación en ellos y las tareas se llevaban a cabo con mucho entusiasmo, recibiendo el respaldo de la comunidad y de las organizaciones. Su radio de acción se amplió: en comedores, con bolsas de cesantes y no participando exclusivamente en torno a los problemas de salud específicos, sino que la salud se 
comenzó a concebir en un sentido integral; se formaron grupos de estimulación, grupos de promoción a la recreación con el objeto fundamental de mejorar las condiciones de vida de la población.

En el año 1978-80 se extendió el programa a toda la zona oeste que comprende las comunas de Cerro Navia, Lo Prado, Quinta Normal, Pudahuel, Estación Central, Maipú y Cerrillos. Se formaron muchos grupos. La Iglesia tenía una organización de comunidades muy amplia.

La zona oeste, división de acuerdo a criterios de la Iglesia, estaba subdividida en decanatos y para cada decanato se destinó una persona que se hizo cargo de la organización de los Equipos de Salud vinculado y coordinado con todos los demás organismos solidarios que allí existían. Existía un organismo de solidaridad en cada decanato que estaba formado por un miembro de la comunidad cristiana, un representante de la comunidad de base, un representante del programa de salud y una de las organizaciones solidarias. En este organismo de solidaridad se planificaban las acciones del decanato, se trabajaba en equipo y simultáneamente se apoyaban las distintas acciones que se desarrollaban en las distintas áreas de solidaridad. Por ende se comenzó a trabajar en la comunidad con una coordinación local de los mismos equipos.

La consolidación de la coordinación de los grupos se planteó como meta en el año 1981.

Alrededor del año 1989 se planteaba la finalización de los Equipos de Salud como Programa de la Iglesia. Se planteó la necesidad de que los grupos de salud se independizaran de la Iglesia y que fueran capaces de ser quienes transmilieran los problemas de salud a la organización poblacional más amplia, al sector oficial de salud y por tanto participar, en alguna medida, en las políticas de salud en su sector. Como una forma de materializar este propósito se hizo necesario que el equipo se abriera a los consultorios del Área Oficial y a los Organismos No Gubernamentales (ONGs) y se trabajó durante el año 1989 con el fin de vincularse como equipo y de vincular a los grupos.

Se trazaron metas y se intentó definir el rol de los grupos de salud en el marco del proceso de democratización del país. Se sostenía la idea de que el lugar de acción del grupo de salud era la organización comunitaria, es decir, la junta vecinal, la unidad vecinal. Sin embargo, los intentos de inserción en las juntas de vecinos tuvieron muchos tropiezos. Estas organizaciones no estaban aún maduras para llevar a cabo un trabajo efectivo. Sin dejar de lado la anterior idea, se planteó otra alternativa que parecía más viable, como era el establecer contacto con los organismos oficiales de salud que existían en las comunas y que eran los consultorios.

Las coordinaciones siguen en la actualidad existiendo con distinto grado de funcionamiento; algunas organizaciones están muy bien montadas, otras han ido desapareciendo.

Por los antecedentes anteriormente expuestos me pareció interesante conocer la experiencia de la participación comunitaria en una organización funcional como es el grupo de salud.

\section{PROPÓSITO Y PASOS DE LA INVESTIGACIÓN}

Este estudio, realizado entre julio y diciembre de 1991, tuvo como propósito recoger algunos testimonios en torno a experiencias en participación comunitaria en salud referida a algunas dimensiones organizacionales de grupos de salud, sus limitaciones y logros en la prosecución de sus objetivos. Dado el carácter exploratorio del estudio y teniendo presente el interés por conocer algo del fenómeno de la participación comunitaria en salud, me pareció conveniente adentrarme en el problema mediante un enfoque metodológico cualitativo. Concretamente me he centrado en el anál isis de opiniones acerca de ciertos factores organizacionales que pudieran estar vinculados con la participación comunitaria en salud en organizaciones funcionales como son los grupos de salud.

La etapa de recolección de información constó de seis salidas a terreno: cuatro en que se 
llevaron a cabo entrevistas a infornantes claves, una en que se hizo una entrevista a un grupo de salud y finalmente una observación no participante en una jornada de reflexión de las actividades realizadas en el año 1991 de los grupos de salud de la zona oeste.

Las cuatro entrevistas individuales fueron hechas a las siguientes personas:

1. Asesora del equipo de atención primaria del Ministerio de Salud, especialista en participación comunitaria en salud. La entrevista a esta informante tuvo básicamente el propósito de indagar acerca de planteamientos en torno a la participación del Programa Oficial Actual.

2. Matrona, que participó como miembro de uno de los Equipos Técnicos que fueron formados por la Vicaría en el año 1976.

3. Enfermera, actualmente miembro en el Programa de Solidaridad del Arzobispado Zona Oeste. Este Programa cuenta con un trabajo de asesoría y acompañamiento a los Grupos de Salud Poblacional.

4. Una pobladora dirigente de la Junta de Vecinos de un Sector de Cerro Navia. Participante actora, desde sus orígenes (1974-75), en un grupo de salud.

Las entrevistas llevadas a cabo fueron inestructuradas, sin una pauta establecida, sin embargo, centrada en los temas que interesaba indagar para responder a los objetivos iniciales de la investigación.

A pesar del número reducido de casos, no fue mi propósito extraer inferencias en torno al comportamiento de las variables en el universo. Más bien me interesaba conocer la versión acerca de la participación, en sus propios términos, en personas que vivenciaron el fenómeno. Por ello la entrevista se llevó a cabo de la manera menos obstrusiva y directiva posible. Mi interés fue más intensivo que extensivo; extraer los supuestos construidos en torno a la participación comunitaria en salud.

\section{RESULTADOS DEL ESTUDIO}

A fin de exponer las distintas visiones de los actores sociales, originadas en su particular inserción y experiencia con el proceso de participación comunitaria en salud, me pareció conveniente organizar este acápite en dos unidades: 1) Una en que se plantean los puntos de vista de los lécnicos que han vivenciado la participación de alguna u otra manera: programando, asesorando y/o acompañando a los actores de los grupos de salud. En este subacápite recogeremos las construcciones de sentido elaboradas por los técnicos (matrona, enfermera y asistente social) en relación a la participación comunitaria en salud, 2) en la segunda unidad se expondrá la experiencia de pobladoras que son micmbros de grupos de salud y que entregan un testimonio algo distinto y enriquecedor dado su papel de actoras directamente afectadas por los problemas de salud que participan en la búsqueda de soluciones a los mismos.

\section{IV.1. La participación comunitaria en Salud vista "desde fuera": las construcciones de sentido de los técnicos}

\subsubsection{El concepto de participación comunitaria en Salud}

La participación en los grupos de salud poblacional es vista como una conducta que se ajusta a las expectativas sociocultural atribuidas al rol de madre.

"En general las mujeres, nosotras vemos que como madres que están pendientes de la salud... llegan al grupo de salud pensando en aprender algo que les pernita ser más eficaces en su casa y zomo están abiertas a atender a sus hijos, están abiertas a otros".

También se postula que la población tiene que lograr organizarse en torno a los problemas de 
salud, como una necesidad que parta de ellos y con una autopercepción favorable acerca de sí mismos, en el sentido de saberse poseedores de habilidades necesarias para participar.

"La salud es una necesidad fundamental de la población, ésta está interesada en participar y debe darse cuenta que tiene habilidades y capacidades que permiten levantar un sistema de salud distinto y alternativo. No antagónico, pero sí puede hacer aportes. La población es capaz de aportar y de hecho siempre lo ha hecho... el problema es que no nos habíamos dado cuenta".

La motivación para participar en Salud específicamente no se la concibe como una imposición, sino como una necesidad que se origina en los mismos actores.

"Es un proceso que parte necesariamente desde las personas, desde las familias, desde las comunidades, desde los niveles locales y una entidad de carácter orientador y político, normativo o encargado de la elaboración programática técnica es mero orientador y asesor que hace un acompañamiento a los procesos que se van a generar desde las realidades particulares locales".

"Yo creo que mientras las personas no tomen conciencia de su responsabilidad ante la salud, es muy poco lo que un Estado tiene que hacer".

"Si lo pensamos desde un punto de vista paternalista, en que tenemos que convencer que lo hagan (que participen), yo creo que es un mito".

Desde el punto de vista de la política de salud del Gobiemo actual la participación de salud es entendida en dos dimensiones: 1) El ámbito extraequipo de salud, en el cual quedan comprendidos el equipo y comunidad. En este contexto los agentes de salud no sólo deben actuar como aplicadores de tecnología médica, sino también como gestores y administradores de las actividades tendientes a solucionar los problemas de salud, 2) la segunda, el de las personas, de las familias, de los grupos y de las organizaciones sociales.

Se reconoce que las condiciones básicas para llevar a cabo una participación activa en la gestión y administración de salud por parte de los agentes de salud no están dadas. Este hecho obviamente tiene un impacto negativo en los propósitos, intereses y motivaciones potenciales, que se pudieran encontrar en la población.

"Si no logramos que haya una estructura de trabajo que tenga instancias de participación que los involucre (a los trabajadores de la salud), que parte de sus necesidades y situación de trabajo no puede haber participación. El diagnóstico con que se inicia el trabajo es tremendamente dramático, por decir lo menos; equipos de salud aislados, encapsulados, deprimidos, burocratizados, en condiciones indignas de trabajo. En su relación con el usuario y con el médico, también es una posición distante".

La situación de los grupos de salud no ha variado sustancialmente con el paso desde un Gobierno en el cual muchos de los espacios de participación se anularon y otros se vieron constreñidos y cuya existencia se mantuvo clandestinamente, hacia un sistema político en que se han abierto las posibilidades de participación, organización a fin de canalizar inquietudes y lograr objetivos. Las motivaciones que dieron lugar al desarrollo de estos grupos en el Gobierno pasado se han visto debilitadas en el presente. Los actores sociales partícipes de estos grupos posiblemente esperan que el cambio de gobierno trajera aparejado una modificación inmediata en su situación de vida.

"El problema está en que la gente creyó que por el hecho de tener democracia iba a cambiar todo y se disolvieron algunos (grupos), otros se desmotivaron. De repente se encuentra que no ha cambiado la situación; que tiene que hacer cola, que tiene dificultad para lograr una buena atención de sus problemas de salud".

"Hay expectativas más allá de la realidad, viven mucho de la historia más que de una realidad concreta de trabajo. Los que aún existen se encuentran en distinto nivel organizacional".

Al parecer los propósitos vertidos en el Programa de Gobierno en el área de la salud 


\section{PARTICIPACIÓN COMUNITARIA EN SALUD: LA EXPERIENCIA...}

apuntando a los principios de Solidaridad, Equidad y Participación ${ }^{1}$ no han logrado aún canalizarse en acciones concretas orientadas a elevar la calidad de vida de la población. No obstante las medidas adoptadas a la fecha para alcanzar los objetivos no han sido pocas:

"El primer año de trabajo del Área de Atención Primaria (del Ministerio de Salud) estuvo centrado en el trabajo de la motivación y la búsqueda del compromiso, pero que partió con una serie de actividades de capacitación y de apoyo a los equipos, tratando de promover y generar un estilo cualitativamente distinto de trabajo al interior (de los Servicios de Salud) que permita reconocerles su condición, sus posibilidades y empezar a tomar parte de un proceso, primero de identificación con la atención primaria, con su trabajo y de allí a participar en un Diagnóstico de la situación de salud del área en que trabajan y por lo tanto que ellos puedan participar en la programación de su trabajo y con perspectivas participativas.

Además en los Consultorios de todo el país se llevaron a cabo Talleres de la Atención Primaria en Salud, con metodologías activo participativas, con monitores que fueron capacitados acá, en el nivel central. Eran personas que habían tenido experiencia en el sector no gubernamental y solidario y del Sector Público en la búsqueda de espacios participativos. Estos talleres tuvieron un efecto multiplicador a nivel de todas las regiones.

Por otro lado se han llevado a cabo discusiones acerca de la propuesta de la estructura para la Atención Primaria, la creación de las Corporaciones de Derecho Público, que va acompañada de un proceso de democratización Municipal, con mecanismos de participación como son los Consejos Comunales de Salud en el cual tenga representación el Sector Solidario, las organizaciones sociales".

Hay un reconocimiento de que la tarea no es fácil ni a corto plazo. Las condiciones de salud como la situación misma de los trabajadores de la Salud ponen en evidencia una crisis del Sector; crisis que no desaparece mediante un decreto de ley.

\subsubsection{Articulación entre actores sociales: comunidad, grupos de Salud y equipos de Salud}

Alrededor del año 1989 los técnicos que asesoraron el proceso de organización y gestión de los grupos de salud consideraron que la Iglesia debería dejar de ser la entidad responsable y coordinadora del trabajo realizado en los grupos. Se planteó la idea de que los grupos de salud tenían que irse desvinculando de la Iglesia y progresivamente, en un escenario sociopolítico

${ }^{1}$ En una ponencia presentada a las Ix Jornadas de Educación para la Salud, el Dr. Roberto Belmar y la Sra. Judith Salinas se refieren a los principios de Solidaridad, Equidad y Participación contenidos en la Estrategia de Atención Primaria del Ministerio de Salud en los siguientes términos: Solidaridad; este nuevo sistema debe establecer la responsabilidad de un Estado solidario que sea capaz de asegurar el acceso a una salud básica a todos los habitantes del país y que se focalice en los grupos prioritarios de marginalidad, ruralidad y aistamiento étnico. Este Estado solidario no sólo debe asegurar el acceso a la salud a los sectores desposeídos, sino además debe regular todo el sistema de salud sin excepción, para asegurar este acceso a todos los sectores con bajos ingresos, aislamiento geográfico, marginación social o expuestos a ricsgos laborales, ambientales y de estilo de vida. Equidad: el sistema de salud debe asegurar cl acceso a las prestaciones básicas de atención primaria y la atención de urgencia a nivel de la estructura hospitalaria del Estado a toda la población del país. Ademís, debe asegurar el acceso a aquellos sectores que están limitados por condiciones como extrema pobreza, ruralidad, marginalidad y a grupos específicamente desprotegidos como por ejemplo los trabajadores no cubiertos por las mutuales de seguridad y minorías étnicas. Participación Social: La participación social no sólo se debe expresar en salud como resultado de una posición idcológica, sino que le es propia a la salud debido a que debe ser la sociedad misma la que reoriente sus hábitos y estilos de vida para prevenir las patologías emergentes como cardivasculares, cánceres, accidentes y daños asociados a alteraciones del medio ambiente o consumo de sustancias nocivas (alcohol, drogas y abaco); al mismo tiempo que la sociedad toda asuma responsabilidades en la mantención de la salud individual y social. Salud: Orientaciones Estratégicas. Dr. Roberto Belmar y Sra. Judith Salinas, 18, 19 y 20 de noviembre 1991, Santiago, Chile. 
distinto, pronto a instaurarse, tendrían que iniciar su vinculación con las organizaciones formales de la población. Se concebía a estos grupos como organismos poblacionales capaces de transmitir los problemas de salud de los pobladores a la organización población más amplia (junta de vecinos, unidad vecinal). Esta articulación entre los grupos de salud y las organizaciones formales reconocidas por los pobladores implica el establecimiento de contactos entre el Equipo Técnico y los Consultorios Oficiales de Salud y las Organizaciones no gubernamentales.

"Hicimos muchos esfuerzos durante todo este año (1989) de vincularnos nosotros, de vincular a las organizaciones con muy buena experiencia en algunas partes. Por primera vez lográbamos entrar como profesionales. Pero también lográbamos que en algunas partes fueran recibidas en grupo".

"Se hizo todo un Programa para buscar, con los mismos grupos, que les tocaba vivir de ahí para adelante. Se trazaron metas a fin de vincularlos a todos con los consultorios. La idea que nosotros teníamos es que el grupo de salud y su lugar de acción cra la organización comunitaria es decir la junta de vecinos, la unidad vecinal y que parte de esa organización es el grupo de salud. Se esperaba incluso que de las organizaciones territoriales surgieran personas interesadas en llevar a cabo un trabajo comunitario en salud".

En la actualidad aún no ha sido posible la concreción de los propósitos del equipo técnico; las juntas de vecinos se encuentran en un proceso de democratización y subsiste una imagen negativa de ellas, herencia del gobierno anterior.

"Las juntas de vecinos no tienen una realidad, una fuerza, una potencia y en algunos casos están completamente politizadas. Algunas no funcionan, otras no están democratizadas aún”.

"Algunos grupos intentaron participar en las organizaciones formales, pero con algunas dificultades. Tampoco estas organizaciones se encontraban maduras en ese momento, porque estos grupos de salud, en los cuales habíamos trabajado por tanto tiempo, pudieran tener un vínculo permanente con ellas y no se murieran".

Toda vez que las condiciones para una articulación eficaz entre grupos de salud y organizaciones forales no estaban dadas, el equipo técnico se propuso trabajar en la búsqueda de articulaciones entre los grupos y consultorios.

"Nos planteamos como otra alternativa, sin descalificar la primera, era permitirles (a los grupos) su cambio funcional; que se contactaran con la organización oficial de salud que existían en las comunas, los consultorios. Esto último con doble vía de apoyo: por un lado que ellos son población y por lo tanto tienen que participar en la organización poblacional y por lo tanto, con un respaldo y vinculados al consultorio de manera de mantener su presencia. Nos dimos cuenta que no somos autosuficientes ninguno, ni las organizaciones, ni nadie".

En los intentos de articulación entre los grupos de salud poblacional y los consultorios hay experiencias exitosas como abortadas. Se piensa que el contacto produce cierto grado de desgaste en ambas organizaciones; por un lado el grupo espera más de lo que el consultorio puede ofrecer y a la vez el equipo de salud del consultorio supone que va a recibir mucho de una población organizada o que también la población puede ser campo de experiencia de los profesionales, suponiendo que su tarea es hacer educación en salud, sin tomar en cuenta que los grupos ya están capacitados.

"Los Consultorios tienen la intención de trabajar en Atención Primaria, pero lamentablemente no han sabido trabajar con la comunidad. Ellos se quejan de que la comunidad los ha defraudado. He conversado con varios directores; ellos tenían muy altas expectativas, yo no sé qué entiendan por trabajar con la comunidad".

Las razones que se esgrimen para explicar esta falta de articulación efectiva entre comunidad Y organismos oficiales de salud apuntan, al parecer, a una descoordinación entre la base (grupos 
de salud y comunidad en general) y las organizaciones formales de la población (Municipalidad, Junta de Vecinos, Unidad Vecinal) y consultorios. Existe un interés manifiesto de parte de los actores sociales de participar en Salud. Sin embargo no hay instancias definidas para llevar a cabo la tarea.

"El consultorio tiene todo el poder de atracción para estimular a la gente a participar, mal que mal a la gente le gusta trabajar en una institución de Salud; como que le da status. Sin embargo, no lo toman en serio. Los grupos se están resistiendo, de alguna u otra forma los han manipulado".

A pesar de que el Área de Salud se ha visto mejorada durante el actual gobierno en términos de ampliación de cobertura, mayor número de atenciones, se evidencia una falta de instancias efectivas de participación comunitaria y de articulación entre comunidad y servicios oficiales de salud.

Entre las estrategias del Programa de Atención Primaria del Ministerio de Salud se estipuló que en una primera etapa de democratización del Área de Salud debía hacerse un reconocimiento al aporte de las Organizaciones no Gubernamentales (ONGs). Además se consideró necesario propender a un contac to y comunicación entre dos mundos alternativos: oficial y no oficial. Este proceso de reconocimiento mutuo no ha estado exento de tropiezos.

"Se está haciendo de forma muy gradual, de forma muy heterogénea y esto tiene que ver con todas las condiciones básicas en que se encuentran cada uno de los consultorios; hay equipos y áreas donde hay un trabajo avanzado, por ejemplo la Comuna de La Florida, donde están funcionando las coordinaciones territoriales de las juntas de vecinos. Éstas trabajan articuladamente con la Corporación de Salud y ésta con los Consultorios. En La Florida se han realizado tres Congresos Comunales de Salud, donde la comuna discute y elabora con todas las organizaciones sociales. En Conchalí pasa algo parecido".

La experiencia de Conchalí y La Florida desgraciadamente no está generalizada. Esto es así porque las historias y características de cada Comuna hacen diferencias.

El Ministerio de Salud tiene una tarea definida en términos de la participación como un fenómeno externo a los Equipos de Salud Oficiales. En esta tarea hay tres líneas de acción definidas:

1. Reconocimiento Público del aporte de las onGs y estimular un proceso de comunicación mutua. "Estos dos mundos alternativos, en una apertura democráticà empiezan a reconocerse, empiezan a reconocer sus aportes mutuos, a reubicar posiciones. Este es un componente fundamental para lograr una gestión participativa, todo esto en cl contex lo de la descentralización en salud, de desarrollo de políticas descentralizadoras y en la perspectiva de configurar las propuestas de los sistemas locales de salud ${ }^{2}$. Concretamente en octubre de 1990 se realiza un Encuentro Nacional cuyo objeto fue apoyar la comunicación regional y local, entre organismos oficiales y no oficiales a fin de promover la formación de redes y espacios de articulación regionales y locales".

\section{Apoyo Técnico Financiero a Proyectos de Acción Directa en Áreas de:}

2.a. Atención Integral de la Salud en Atención Primaria

2.b. Promoción de la Salud

2.c. Investigación Operativa

\footnotetext{
${ }^{2}$ "Un sistema local de salud se define como un conjunto interrelacionado de recursos de salud sectoriales e ntersectoriales responsable por la salud de una población y un ambiente en un territorio definido. A través del sistema bcal de salud se coordinan los recursos del sector salud y otros, se facilita la participación social, se reorienta el sistema Tacional de salud y el desarrollo del país". En Participación en Salud Lecciones y Desafios de Teresa Marshall, Margarita ऽáez y Judith Salinas. Editado por la Corporación de Salud y Políticas sociales (CORSAPS) mayo 1991, pág. 21.
} 
REVISTA DE SOCIOLOGIA

3. Promoción de Acciones que Favorezcan el Trabajo Comunitario desde los Equipos de Atención Primaria en Salud. Por ejemplo que los equipos de salud elaboren diagnósticos participativos de salud.

\section{IV.2. La participación comunitaria en salud desde la perspectiva de las pobladoras}

\subsubsection{Significado de la Participación Comunitaria en Salud}

Para algunas pobladoras su concepción de lo que es participación va ligado a la idea de que es el ser humano que por sí mismo debiera llevar a cabo tareas para poder alcanzar los objetivos propuestos.

"Nosotros somos gestores de nuestra propia vida y debemos participar en todo aquellos que nos va, de alguna u otra forma, afectando. Pero no se da".

Hay un reconocimiento de que hay mujeres que participan en los grupos de salud para lograr ciertos objetivos concretos. La motivación para participar no está exenta de interés individual; si no hay una necesidad no se participa y se participa cuando se cree que mediante este compromiso se podrán satisfacer necesidades sentidas.

"Participan en las asambleas cuando quieren que se les dé algo. Entra el oportunismo. Antes y ahora se justifica por la situación económica de las socias. Por la falta de plata participan más solidariamente. Pero, éstas son una mínima parte del grupo".

Las pobladoras que participan teniendo como propósito la satisfacción de una necesidad individual conforman el grupo de aquéllas que se alejan una vez que logran su objetivo.

"La gente le interesa (participar) cuando le da algo. Esa gente es la que se retira con el tiempo".

Muchas de las mujeres pobladoras que han participado desde la formación de los grupos y se han mantenido en esta tarea están motivadas por fines solidarios, de apoyo a los más necesitados.

"Tenemos que aprender para entregar salud, dentro de nuestras posibilidades, a la comunidad".

"Es bonito que una persona que tenga conocimientos que lo dé".

"Las que tienen cariño a la organización van a seguir y las que no, no".

"Yo he permanecido por lo cue significa estar en una organización solidaria y comunitaria".

"Pienso que hay que dar algo, la gente que no se organiza, no puede hacerlo".

"Nos hemos mantenido por nuestra motivación para transmitir conocimientos sobre enfermedades a la gente joven".

Las afirmaciones de las mujeres pobladoras ponen en evidencia que ellas sostienen un concepto de participación bien definido: varticipar es organizarse, vincularse a la comunidad para entregar conocimientos que sirven para ayudar a otros a resolver sus problemas. Es importante enfatizar la dimensión de entrega orienta la a la ayuda de otros que surge de su discurso.

"Todo lo que sea para que la gente sepa educarse. Lo que sea para bien de sus hijos, la gente lo va a aceptar".

No obstante, la participación como un quehacer, que en alguna medida hace posible alcanzar soluciones a los problemas es una categcría ausente en la conciencia de muchas pobladoras. Esta carencia es atribuida a la falta de aprendizaje en participación y a la enajenación de las pobladoras producto de su status social discriminado. Esta situación es especialmente evidente y marcada en los sectores populares.

"Hay una falta de educación muy grande a nivel de la mujer; a la mujer se le ha tratado mucho de adormecer ¿con qué? con los medios de comunicación, con las teleseries, con las comedias. Esto es prioridad para las mujeres, no su realidad. Con eso adormecen sus problemas, se evaden de su propia realidad y penetrar a ése cuesta mucho. Es decir educarlas, formarlas cuesta mucho. 
Es más grande la presión de los medios de comunicación de masas. Tú ves que la labor que podemos hacer nosotras; entregar nuestro horario, una o dos horas a la semana con distintas personas. Entonces cuesta llegar. Lo otro es más cómodo; tú ves tu comedia y te quedas tranquila, duermes siesta y ves la realidad de tu casa, nada más".

\subsubsection{La Planificación de las Actividades}

Los grupos desde sus comienzos, en los cuales contaron con técnicos que contribuyeron a su formación y organización, han desarrollado una planificación de sus actividades. La planificación se realiza en base al análisis de las tareas cumplidas y necesidades insatisfechas, es decir, los grupos tienen espacios de encuentros en los cuales discuten las actividades del período concluido y evalúan logros y fracasos. De esa evaluación se elabora un programa en que se definen las actividades para el período de trabajo que está por iniciarse.

"Participamos siendo formados, primero y nos ayudaron (los técnicos) a planificarnos, pero después siendo más autónomos íbamos planificando de acuerdo a las realidades de nuestras Comunas. En base a nuestras necesidades elaborábamos un programa, ya sea semestral o anual. Los primeros años la planificación la hacíamos en una salida de tres a cuatro días fuera de Santiago. La jornada consistía en dos etapas. Una era de trabajo y otra de recreación; toda la mañana se trabajaba y en la tarde se hacía recreación, se aprovechaba la playa".

"Al grupo le decimos que lleve propuestas de qué quieren que se haga durante el año. Nos dividimos en cuatro grupos, lo que propone la dirección junto con lo que aparece más reiteradamente, va demostrando prioridad. Las actividades se planifican de marzo a agosto. Si cumplimos los objetivos, seguimos de agosto a diciembre. Por lo general cumplimos todo el Programa".

\subsubsection{Sentimiento del "Nosotros" y Logro de Autonomía}

Las experiencias adquiridas durante el proceso de formación iniciado en los años 1974-1975, la capacitación recibida de los técnicos asesores y todo el trabajo llevado a cabo por los grupos, con altos y bajos, han desarrollado en ellos la habilidad de autogestión. Incluso las pobladoras concuerdan en que la percepción de "sus" problemas no necesariamente coinciden con la visión "desde afuera" que puede sostener el técnico.

"Porque es distinto el asesor que viene de afuera a nosotros que conocemos nuestra propia realidad".

Las pobladoras sostienen que algunas de las causas de quiebres que se han presentado en la organización de estos grupos es la presencia e ingerencia de personas ajenas a los mismos y el debilitamiento de la estructura coordinadora zonal. Esta coordinadora zonal se fornó después de algunos años de funcionamiento de los grupos, a fin de agruparlos y organizar de manera conjunta las actividades de todos los grupos pertenecientes a la misma zona ${ }^{3}$.

"Son otras personas que no han tenido la experiencia de nosotras las que programan y disponen las platas".

"El trabajo lo generó la Iglesia, en base a la necesidad de la población. Hasta allí éramos muy amigas, pero después empezamos a tomar conciencia, a empezar a educarnos. Se creó una brecha con aquellas personas que estaban 'más pegadas' a los padres (sacerdotes). En Pudahuel hay gente muy carismática, gente de edad, de rezar el rosario y salió una generación joven que no creía que rezando se iba a traer comida a los niños".

${ }^{3}$ Cada coordinadora zonal corresponde a la división zonal de la Iglesia: zona Oeste, Este, Norte y Sur. 
La formación de estos grupos ocurre en un momento histórico sociopolítico de nuestro país, respuesta a la necesidad de proteger y dar salud a personas víctimas de la represión. Por ende para las pobladoras miembros de estos grupos de Salud, el estímulo por participar en estas organizaciones desborda la búsqueda de soluciones a sus problemas de salud y su quehacer en ellas implica asumir que la salud no es mero estado opuesto a la enfermedad, sino que supone una concepción integral de la salud que pasa necesariamente por una mejora en las condiciones y estilos de vida de las personas. Esto implicó que algunos grupos en los cuales existe una mayor conciencia acerca de sus precarias condiciones de vida se dieran a la tarea de capacitar en una construcción social de la salud desde una perspectiva holística.

"La capacitación consistía en saber lo que es la salud misma. Uno cree que estando el cuerpo sano está bien y no es así; la salud no consiste en eso, la salud consiste en que tienen que tener vivienda, educación, recreación, alimentación, un montón de cosas, es algo integral".

\subsubsection{Capacitación en Salud}

La Capacitación y Educación para la Salud, en los primeros años en los cuales estos grupos se organizaron y se fueron abriendo espacios en sus respectivas poblaciones estaba bajo la responsabilidad de los Equipos Técnicos de Salud.

"Recibimos cursos de primeros auxilios porque habían carencias muy grandes de médicos, de horas asistenciales. Para responder a las necesidades de salud en nuestra población ayudábamos nosotras con curaciones, en tratamiento de inyectables, en prevención de diarrea. O sea lo más importante era ayudar a prevenir a que no se declararan las enfermedades. Esta capacitación duraba un año, año y medio o dos".

La capacitación recibida por las pobladoras miembros de los grupos de salud tenía un efecto multiplicador.

"Cada cual veía cuál era su vocación, si era trabajo asistencial o si se encontraba capacitada para poder salir a educar a las pobladoras. Si se encontraba capaz, reunía a un grupo de amigas y empezaba a formarlas en la educación que había recibido, apoyada por los asesores que venían de vez en cuando y veían que se estuviera desarrollando bien".

\subsubsection{Liderazgo al interior de las Organizaciones}

El ejercicio del liderazgo al interior de los grupos de salud no ha generado problemas en la dinámica de estas organizaciones. Las mujeres que han tenido la responsabilidad de dirigir los grupos han sido democráticamente elegidas. Aquéllas que no han respondido satisfactoriamente a las expectativas del grupo han tenido que abandonar voluntariamente la presencia del equipo.

"Se elige a la directiva porque lo hacen bien; ha de ser una persona solidaria, participar y además tiene que tener tiempo".

"Hay personas que quieren ser caudillos, ser los únicos. Eso va en el grupo en sí; si va a saber bajarle el moño a la persona. Las personas van a saber controlarlo. Cada dos años cambia la directiva, nadie que se pegue".

"Por la Directiva se proporciona gente que trabaja que están en la cuestión en el año. Que trabaja con auge. Se propone al candidato en la asamblea y se vota por voto secreto o mano alzada".

"Los dirigentes nunca fueron autónomos, o sea, todo lo que hacen tiene que ser consultado en la asamblea. La asamblea es la que tiene el poder decisivo para hacer las cosas".

Me parece pertinente presentar a continuación el testimonio de una mujer que tiene una larga 
trayectoria como miembro fundadora de grupos de salud y en la actualidad ocupa el cargo de dirigente de una junta de vecinos de la población en que vive. Su testimonio sirve para aclarar las diferencias que se perciben entre los dirigentes de las Juntas de Vecinos y aquéllos de los grupos de salud:

"En salud es gente con una conciencia; gente que está unida porque hay un problema y saben que la única manera de solucionarlo es uniéndose, educándose, buscando objetivos para poder llevar adelante su problema.

En la Junta de Vecinos es completamente distinto; tú vas a una elección; los pobladores te eligen. De hecho tú tienes que tener una imagen poblacional para que los pobladores puedan votar por ti.

Hay problemas, pero no toda la gente está consciente de ellos. Creen que el dirigente es un empleado que tiene que solucionar sus problemas, no importa cómo, ni a la hora que sea. Pierdes tu privacidad; pueden ser las tres o cuatro de la mañana y tú tienes que levantarte y aparte que tú no recibes sueldo, es completamente voluntario el trabajo.

Pero no entienden que si ellos se organizan y ven en conjunto sus problemas, se puede avanzar mejor y no tirarle el problema al dirigente, que el dirigente solo lo resuelva con el Directorio.

Es completamente distinto el trabajo (respecto al grupo de salud). Además que con el grupo de salud se trabaja con no más allá de 50 personas. En cambio con la Junta de Vecinos se trabaja con 600 socios y 600 opiniones distintas y la población es cuatro o cinco veces más grande que eso. Están todos los que no son socios, pero no se puede atender sólo a los socios, sino a toda la gente de tu población".

\subsubsection{La Praxis de los Grupos de Salud en la Actualidad}

En el presente estos grupos han visto disminuido el número de miembros tomando en cuenta que desde su surgimiento, hace ya cerca de dieciocho años, han experimentado cambios directamente asociados a la realidad histórica del país. No obstante lo anterior, los que han permanecido a lo largo del tiempo realizan actividades bien delimitadas a fin de ayudar en la solución de los problemas de salud en su comunidad.

"Uno de los trabajos es el trabajo asistencial a enfermos que no tienen los medios suficientes para llegar al Consultorio por ser indigentes o porque son ancianos postrados en cama, que hay que tener vehículo para sacarlos. Entonces el trabajo mío es ir a sus casas, atenderlos, hacerles su tratamiento".

"Establecer enlaces entre los enfermos con la asistente social del Consultorio o derivarlos a algún Servicio del Consultorio. Es por medio de la asistente social y por la maternidad que tenemos el camino más fluido para llegar allá".

"Pasar vidcos en la población sobre algo relacionado con la Salud. Cada una tiene la tarea de traer dos o tres personas y poner afiches en partes concurridas para avisar del video que vamos a pasar".

Los grupos cuentan con un calendario de reuniones y programas. Éstos se definen de acuerdo a sus particulares necesidades. Aun cuando los encuentros no son muy frecuentes, sino cada dos o tres meses, igualmente cada miembro continúa realizando su trabajo con la gente.

"Basta con que haya una necesidad, una emergencia o por algo tenemos que encontrarnos, basta con que le avisemos a una persona y así sencillamente, nos vamos avisando unos a otros y logramos comunicarnos". 


\section{RESUMEN Y CONCLUSIONES DEL ESTUDIO}

En el transcurso del presente trabajo hemos intentado dar cuenta de las experiencias en torno a la participación comunitaria en Salud de personas que han intervenido en la organización y gestión de las actividades de estos grupos y de personas que como actores que enfrentan problemas de salud, están comprometidas en la búsqueda de soluciones a los mismos.

La participación en salud es concebida como una tarea que es estimulante para la acción de la población que se compromete a ella, especialmente cuando la participación no es una actividad impuesta "desde afuera", sino que surge de las necesidades de los mismos actores. Se considera que la población está capacitada para ser gestora del proceso de participar. Al respecto, se postula de que la inexistencia de una conciencia de que se tiene la capacidad de participar no puede suponerse que será un objeto posible de lograr, mediante una propuesta que se origine en personas que perciben la participación desde una óptica distinta a la de los actores potenciales participantes del proceso.

Por otro lado, se reconoce que es urgente la creación de mecanismos formales que generen los espacios de participación comunitaria en Salud. La existencia de estos mecanismos formales supone una modificación de la estructura y proceso en el trabajo realizado al interior de los Servicios Públicos de Salud a fin de crear instancias de encuentro y de discusión de los problemas de salud entre agentes de salud y la población. La población, el consultorio y las organizaciones formales de la comunidad (municipalidad, junta de vecinos, unidades vecinales, etc.) requieren lograr un estado de articulación de modo de lograr una participación comunitaria eficiente.

Por parte de las actoras sociales del proceso hay un reconocimiento de que algunas pobladoras ingresan principalmente a los grupos de salud por un interés personal, más que por el propósito de ayudar en la solución de los problemas de Salud de la población. No obstante se produce un decantamiento con el transcurso del tiempo y sólo aquellas mujeres con un interés solidario mantienen su membresía.

El nivel de conciencia y la actitud crítica presentes en el análisis de la desmotivación por participar en los grupos de salud es realmente destacable, se cree que la falta de educación en participación y el estado de enajenación del ser mujer serían las determinantes de la falta de motivación. Por otro lado se asume que la participación en salud implica la búsqueda de soluciones no exclusivamente a los problemas de enfermedad, dado que el restablecimiento de la salud, es sólo una de las varias dimensiones de lo que es la salud.

Desde sus orígenes ha existido en los grupos un trabajo planificado y coordinado. Al comienzo fue dirigido por los técnicos y la misma capacitación fue estimulando en ellos la necesidad de autogestión. En la actualidad los técnicos hacen un trabajo principalmente de acompañamiento en el proceso.

No se han presentado problemas de liderazgo al interior de los grupos. Probablemente las características democráticas en la que se produce el surgimiento del líder explica que este rol organizacional no ha sido fuente de conflictos.

\section{BIBLIOGRAFÍA CONSULTADA}

ASTORGa: El papel de los Grupos de Salud. Notas para un debate. En revista Hechos Humanos.

R. Belmar y J. Salinas: Atención Primaria y Participación Comunitaria en Salud: Orientaciones Estratégicas. En IX Jornada de Educación para la Salud. 18/19/20 noviembre, 1991. Santiago, Chile.

Hevia, P.: La Estrategia de Atención Primaria de Salud. Base para una propuesta de organizaciones de sistemas comunales de Salud en Chile. En Enfoques en atención primaria año 4, № 2, 1989, pp. $20-26$.

Hevia, P.: Los desafíos de la participación social en el Nivel Local de la Salud. Enfoques en Atención Primaria, Año 4, NN 1,1989 , pp. $25-30$. 


\section{PARTICIPACIÓN COMUNITARIA EN SALUD: LA EXPERIENCIA...}

T. Marshall, M. SÁez y J. Salinas: Participación en Salud: Lecciones y Desafíos. Corporación de Salud y Politicas Sociales. CORSAPS. 1991, Chile.

MINISTERIO DE SALUD DIVISIÓN DE PROGRAMACIÓN DEPARTAMENTO DE ATENCIÓN PRIMARIA. Seminario: Políticas de Salud y Organizaciones no Gubernamentales. Santiago de Chile, 9 y 10 de octubre, 1990.

OMS, UNICEF: Atención Primaria en Salud. ALMA ATA, 1978.

OMS, CIPHE: La Educación para la Salud. 1985, № 1.

Pedersen, D.: Guía para la Evaluación de la Participación de la Comunidad en los Servicios de Salud, Washington, D.C. E.U.A. 1988.

M.A. TONON: Concepts in Community Participation: a case of sanitary change in a guatemalan village. En International Journal of Health Education Supplement to Vol. XXIII, Issue № 4, Oct-Dec. 1980.

A. UGaLdE: Ideological Dimensions of Community Participation in Latin American Health Programs. En Social Science and Medicine. Vol. 21, № 1, 1985. 